QUARTERLY OF APPLIED MATHEMATICS

VOLUME LXIV, NUMBER 4

DECEMBER 2006, PAGES 593-604

S $0033-569 X(06) 01020-9$

Article electronically published on September 11, 2006

\title{
PERISTALTIC TRANSPORT OF A HERSCHEL-BULKLEY FLUID IN CONTACT WITH A NEWTONIAN FLUID
}

\author{
$\mathrm{BY}$ \\ K. VAJRAVELU (Department of Mathematics, University of Central Florida, Orlando, Florida \\ 32816), \\ S. SREENADH (Department of Mathematics, Sri Venkateswara University, Tirupati 517502, India), \\ AND \\ V. RAMESH BABU (Department of Mathematics, S. V. Arts College, Tirupati, India)
}

\begin{abstract}
Peristaltic transport of Herschel-Bulkley fluid in contact with a Newtonian fluid in a channel is investigated for its various applications to flows with physiological fluids (blood, chyme, intrauterine fluid, etc.). The primary application is when blood flows through small vessels; blood has a peripheral layer of plasma and a core region of suspension of all the erythrocytes. That is, in the modeling of blood flow, one needs to consider the core region consisting of a yield stress fluid and the peripheral region consisting of a Newtonian fluid. Peristaltic pumping of a yield stress fluid in contact with a Newtonian fluid has not previously been studied in detail. Our goal is to initiate such a study. The Herschel-Bulkley fluid model considered here reduces to the power law model in the absence of yield stress.

The stream function, the velocity field, and the equation of the interface are obtained and discussed. When the yield stress $\tau_{0} \rightarrow 0$ and when the index $n=1$, our results agree with those of Brasseur et al. (J. Fluid Mech. 174 (1987), 495) for peristaltic transport of the Newtonian fluid. It is observed that for a given flux $\bar{Q}$ the pressure rise $\Delta p$ increases with an increase in the amplitude ratio $\phi$. Furthermore, the results obtained for the flow characteristics reveal many interesting behaviors that warrant further study of the peristaltic transport models with two immiscible physiological fluids.
\end{abstract}

1. Introduction. Physiological fluids in animal and human bodies are, in general, pumped by the continuous periodic muscular oscillations of the ducts. These oscillations are presumed to be caused by the progressive transverse contraction waves that propagate along the walls of the ducts. Peristalsis is the mechanism of the fluid transport that occurs generally from a region of lower pressure to higher pressure when a progressive wave of area contraction and expansion travels along the flexible wall of the tube. Peristaltic

Received June 21, 2004.

2000 Mathematics Subject Classification. Primary 34B15, 92C10.

Key words and phrases. Peristaltic transport, Herschel-Bulkley fluid, Newtonian fluid, volume flow rate. 
flow occurs widely in the functioning of the ureter, food mixing, and chyme movement in the intestine. It also occurs in the movement of eggs in the fallopian tube, the transport of the spermatozoa in the cervical canal, transport of bile in the bile duct, transport of cilia, and circulation of blood in small blood vessels. There are many other important applications of this principle such as the design of roller pumps, which are useful in pumping fluids without contamination due to contact with the pumping machinery.

In 1966, Latham [1 made an experimental study of the mechanics of peristaltic transport. The results of the experiments were found to be in good agreement with the theoretical results of Shapiro 2. Based on this experimental work, Burns and Parkes [3] studied the peristaltic motion of a viscous fluid through a pipe and a channel by considering sinusoidal variations at the walls. Shapiro et al. 4, in 1969 analyzed peristaltic pumping with long wavelengths at low Reynolds number. The small Reynolds number assumption of Shapiro et al. [4 was endorsed by Jaffrin [5] who extended the analysis by considering the higher-order terms to include cases where the Reynolds number was higher. Barton and Raynor [6] studied peristaltic flow in tubes using long wave approximation. Barton and Raynor also analyzed the case for a low Reynolds number. Peristaltic waves in circular cylindrical tubes were analyzed by Yin and Fung [7, and a complete review of peristaltic transport is given by Jaffrin and Shapiro [8].

In physiological peristalsis, the pumping fluid may be considered as a Newtonian or a non-Newtonian fluid. Kapur 9] suggested several mathematical models for the physiological flows. Among these some models deal with Newtonian fluids and others with non-Newtonian fluids. Kapur made theoretical investigations of blood flows by considering blood as a Newtonian as well as a non-Newtonian fluid. Blair et al. [10] reported that blood obeys Casson's model for moderate shear rate flows. Furthermore, they observed that there is no difference between Casson's and Herschel-Bulkley's plots over the range where Casson's plot is valid for blood. In particular they suggested that the Herschel-Bulkley model more closely explains the phenomena for cow's blood than Casson's model. Chaturani and Samy [11] discussed blood flow through a stenosed artery by considering blood as a Herschel-Bulkley fluid. Peristaltic pumping of viscoelastic fluids was investigated by Bohme and Friedrich [12. Shehawey and Mekheimer [13 discussed couple-stresses in peristaltic transport of fluids. Peristaltic transport of a physiologicalfluid in a tapered channel is studied by Eytan et al. [14, and this model is applied to explain embryo transport within the uterine cavity.

We know, of course, that a Herschel-Bulkley fluid is a semisolid rather than an actual fluid. We know by definition, a fluid cannot support a shear stress. This implies that materials that have a "yield-stress" are not fluids - such materials are referred to as a Bingham fluid. A detailed discussion of the inappropriateness of the use of such models for fluids is discussed in the recent review paper by Krishnan and Rajagopal 15. While such materials might not be fluids, there is value in studying them as they give some idea of the behavior of fluids of interest under certain limits.

Among models of semisolids the Herschel-Bulkley model is preferable because it describes blood behavior very closely and because the Newtonian, Bingham, and power-law models can be derived as special cases. Furthermore, Herschel-Bulkley fluids describe 
very well material flows with a nonlinear stress-strain relationship either as a shearthickening or a shear-thinning one. Since the shear-thinning and shear-thickening fluids play an important role in biomedical engineering, Málek and his co-workers studied thoroughly several issues related to these fluids (for rigorous mathematical issues see Málek et al. [16; for global existence results see Málek et al. [17] and for regularity and stability results see Málek et al. [18]). Some examples of fluids behaving in this manner include food products, pharmaceutical products, slurries, polymeric solutions, and semisolid materials (for details see Alexandrou et al. [19]).

There are numerous examples of peristaltic motion. Most living systems involve movement of physiological fluids in response to mechanical forces, for example, mobility of the spermatozoa and ova, propulsion of bacteria and protozoa, blood flow through circulatory systems of mammals. The characteristics of these systems are studied through suitable mathematical models of the systems using the principles of biofluid mechanics. It has been suggested that the peristalsis may be associated with the vasomotion of small blood vessels. Biomedical instruments such as heart-lung machines work using this mechanism. Mechanical devices such as finger pumps apply peristalsis when pumping blood and other bio-fluids. The peristaltic pumping of a toxic liquid is used in the nuclear industry to avoid environmental contamination.

The viscosity near the wall of the duct has been found to be different than that in the central region for many biological systems. Taking this fact into consideration Shukla et al. 20, using Stokes approximations, studied two layered peristaltic flows through tubes and channels. The results are applied to intestinal flows and the flows in the ducts efferentes of the male reproductive tract. The effect of a peripheral layer of different viscosity on peristaltic pumping with Newtonian fluid is discussed by Brasseur et al. 21]. Peristaltic motion of two immiscible fluids in a tube is investigated by Rao and Usha 22. Usha and Rao 23. discussed the peristaltic pumping of two layered power-law fluids in a tube. Most of the tubular organs in the living body contain a thick coating of mucus secreted at the inner surface of the tube walls, which serves as an excellent lubricant and protectant for the tube walls. The composition and the rheology of the gastric mucus have a significant effect on the chyme transport in the gastrointestinal tract. Experiments show that the rheological nature of the mucus can vary from a weak, viscoelastic gel to a shear thinning or Newtonian fluid depending on the concentration of mucin. For example, the rat gastric mucus is observed to show a shear-thinning behavior with apparent viscosity $7.8 \times 10^{4} \pm 1.1 \times 10^{5} \mathrm{~Pa} \cdot \mathrm{s}$ (Zahm et al. 24]). Hence, the study of gastrointestinal fluid mechanics is incomplete without understanding the effect of rheology of mucus as well as that of the chyme on peristaltic flow.

Motivated by these studies and their various applications, the peristaltic transport of two fluids in a channel is investigated. The fluid in the core layer is described by the Herschel-Bulkley model and the fluid in the peripheral layer is considered to be a Newtonian fluid. The interface, which is also a streamline, is determined from a nonlinear equation. The velocity, the stream function, and the pressure rise are obtained. Some deductions are made, and the results agree with those of earlier works. 
2. Basic equations. The basic equations governing the flow of an incompressible Navier-Stokes fluid are the field equations

$$
\operatorname{div} \mathbf{V}=0, \quad \operatorname{div} \sigma+\rho \mathbf{f}=\rho \frac{d \mathbf{V}}{d t},
$$

where $\mathbf{V}$ is the velocity, $\mathbf{f}$ the body force per unit mass, $\rho$ the density, and $d / d t$ the material time derivative. $\sigma$ is the Cauchy stress defined by

$$
\begin{aligned}
\sigma & =-p \mathbf{I}+\mathbf{T}, \\
\mathbf{T} & =2 \mu \mathbf{D}+\mathbf{S}, \\
\mathbf{S} & =2 \eta \mathbf{D},
\end{aligned}
$$

where $\mathbf{D}$ is the symmetric part of the velocity gradient, that is,

$$
\mathbf{D}=\frac{1}{2}\left[\mathbf{L}+\mathbf{L}^{T}\right], \quad \mathbf{L}=\operatorname{grad} \mathbf{V} .
$$

Also, $-p \mathbf{I}$ denotes the indeterminate part of the stress due to the constraint of incompressibility; $\mu$ and $\eta$ are viscosities.

The Herschel-Bulkley model combines the effects of Bingham and the power-law behavior in a fluid. For low strain rates $\left(\dot{\gamma}<\tau_{0} / \mu_{0}\right)$, the "rigid" material acts like a very viscous fluid with viscosity $\mu_{0}$. As the strain rate increases and the yield stress threshold, $\tau_{0}$, is passed, the fluid behavior is described by a power law

$$
\eta=\frac{\tau_{0}+k\left[\dot{\gamma}^{n}-\left(\tau_{0} / \mu_{0}\right)^{n}\right]}{\dot{\gamma}},
$$

where $k$ is the consistency factor and $n$ is the power-law index.

3. Formulation and solution of the problem. Consider the peristaltic transport of a physiological fluid consisting of two immiscible and incompressible fluids of different viscosities occupying a channel of half-width $a$. The core fluid is a Herschel-Bulkley fluid with viscosity $\mu_{1}$ while the peripheral layer is a Newtonian fluid with viscosity $\mu_{2}$. The wall deformation due to the propagation of an infinite train of peristaltic waves is given by

$$
Y=H(X, t)=a+b \sin \frac{2 \pi}{\lambda}(X-c t),
$$

where $b$ is the amplitude, $\lambda$ is the wavelength and $c$ is the wave speed (for details see Figure 1). The subsequent deformation of the interface separating the core and the peripheral layer is denoted by $Y=H_{1}(X, t)$, which is not known a priori.

Two facts follow from the assumption that the channel length is an integral multiple of the wavelength $\lambda$. First, the pressure difference across the ends of the channel is a constant and the periodicity of the interface is the same as that of the peristaltic wave. Secondly, the flow becomes steady in the wave frame $(x, y)$ moving with the velocity $c$ away from the fixed frame $(X, Y)$ called the laboratory frame. The transformation 


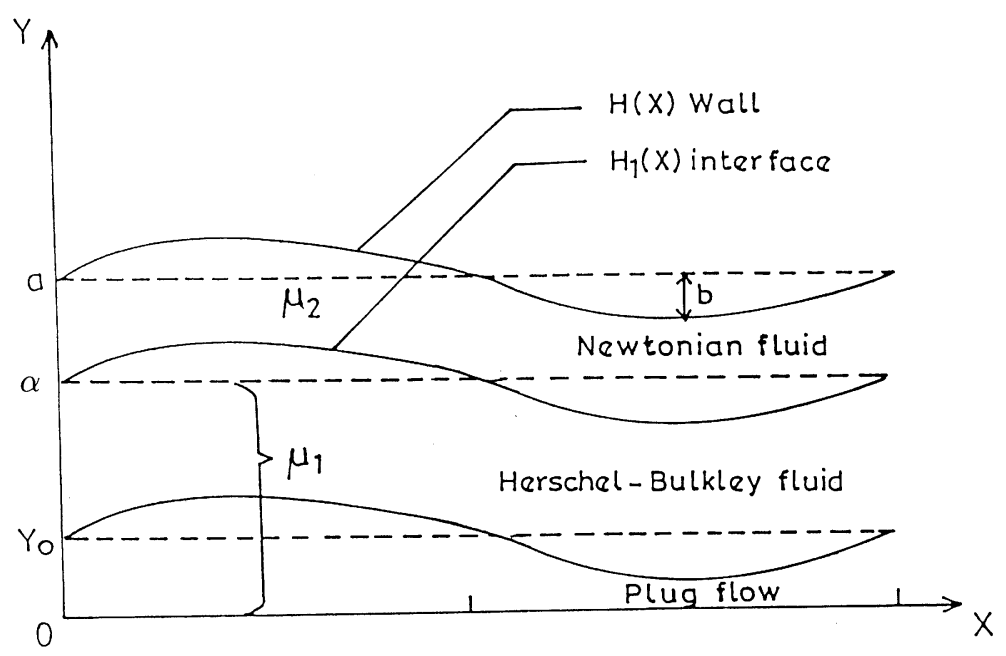

Fig. 1. Physical model

between these two frames is given by

$$
\begin{aligned}
x=X & -c t, \quad y=Y \\
u(x, y) & =U(X-c t, Y)-c \\
v(x, y) & =V(X-c t, Y) \\
p(x) & =P(X, t) \\
\psi & =\Psi-Y
\end{aligned}
$$

where $\psi$ and $\Psi$ are the stream functions in the wave and laboratory frames, respectively, using the following nondimensional quantities:

$$
\left.\begin{array}{l}
\bar{x}=\frac{x}{\lambda} ; \quad \bar{y}=\frac{y}{a} ; \quad \bar{h}=\frac{h}{a} ; \quad \bar{t}=\frac{c t}{\lambda} \\
\phi=\frac{b}{a} ; \quad \bar{\tau}_{0}=\frac{\tau_{0}}{\mu\left(\frac{c}{a}\right)^{n}} ; \quad \bar{p}=\frac{a^{n+1}}{\lambda \mu c^{n}} p \\
\Psi^{(i)}=\frac{\psi^{(i)}}{a c} ; \quad \bar{q}=\frac{q}{a c} ; \quad \bar{F}=\frac{F a}{\mu_{1} \lambda c} \\
\bar{u}^{(i)}=\frac{u^{(i)}}{c}=\frac{\partial \Psi^{(i)}}{\partial \bar{y}} ; \quad \bar{\nu}^{(i)}=\frac{\nu^{(i)} \lambda}{a c}=\frac{\partial \Psi^{(i)}}{\partial \bar{x}} \\
\quad \bar{\mu}=1, \quad 0 \leq \bar{y} \leq \bar{h}_{1}, \\
\bar{\mu}=\mu\left(=\frac{\mu_{2}}{\mu_{1}}\right), \quad \bar{h}_{1} \leq \bar{y} \leq \bar{h}
\end{array}\right\}
$$


where $\bar{u}^{(i)}$ and $\bar{v}^{(i)}$ are the $\bar{x}$ and $\bar{y}$ components of velocities in the wave frame. Under the lubrication approach, the equations governing the motion become (ignoring the bars)

$$
\begin{gathered}
\frac{\partial}{\partial y}\left[\tau_{0}+\mu_{1}\left(\frac{-\partial^{2} \psi^{(1)}}{\partial y^{2}}\right)^{n}\right]=-\frac{\partial p}{\partial x} \\
0=\frac{\partial p}{\partial y}
\end{gathered}
$$

and

$$
\frac{\partial}{\partial y}\left[\mu_{2}\left(\frac{-\partial^{2} \psi^{(2)}}{\partial y^{2}}\right)\right]=-\frac{\partial p}{\partial x}
$$

The dimensionless boundary conditions are

$$
\begin{aligned}
& \Psi_{p}^{(1)}=0 \quad \text { at } \quad y=0 \text {, } \\
& \tau_{y x}=0 \quad \text { at } y=0 \text {, } \\
& \Psi^{(2)}=q=\text { constant } \quad \text { at } \quad y=h \text {, } \\
& \Psi^{(1)}=q_{1}=\text { constant } \quad \text { at } y=h_{1}, \\
& \Psi_{y}^{(2)}=-1 \quad \text { at } y=h,
\end{aligned}
$$

where $q$ and $q_{1}$ are the total and the core fluxes respectively across any cross section in the wave frame. Furthermore, the velocity and the shear stress are continuous across the interface. The peripheral-layer flux is $q_{2}=q-q_{1}$. We infer from the incompressibility of the fluids and the lubrication theory that $q, q_{1}$ and $q_{2}$ are independent of $x$. The average nondimensional volume flow rate $\bar{Q}$ over one period $T\left(=\frac{\lambda}{c}\right)$ of the peristaltic wave is defined as

$$
\begin{aligned}
\bar{Q} & =\frac{1}{T} \int_{0}^{T} \int_{0}^{h}(u+1) d y d t \\
& =q+1
\end{aligned}
$$

The stream function is obtained using the boundary conditions (3.7) to (3.11) together with the boundary conditions at the ends of the channel given by specifying $\bar{Q}$ or the pressure difference $\Delta p$ across one wavelength.

On solving equations (3.4)-(3.6) together with the boundary conditions (3.7)-(3.11) and

$$
\psi^{(1)}=\Psi_{p}^{(1)} \quad \text { at } \quad y=y_{0},
$$

where $y_{0}$ is the upper limit of the plug flow region and $\psi_{p}^{(1)}$ is the stream function in the plug flow region, we obtain the stream function in the core (plug flow region and 
non-plug flow region) and peripheral layer. This is

$$
\begin{aligned}
& \psi_{p}^{(1)}=-y+\frac{P^{k} y}{k+1}\left[\left(h_{1}-\frac{\tau_{0}}{P}\right)^{k+1}-\left(y_{0}-\frac{\tau_{0}}{P}\right)^{k+1}\right] \\
& +\frac{P y}{2 \mu}\left(h^{2}-h_{1}^{2}\right) \text { for } \quad 0 \leq y \leq y_{0}, \\
& \psi^{(1)}=-y+\frac{P y}{2 \mu}\left(h^{2}-h_{1}^{2}\right) \\
& +\frac{P^{k}}{k+1}\left[y\left(h_{1}-\frac{\tau_{0}}{P}\right)^{k+1}-\frac{\left(y_{0}-\frac{\tau_{0}}{P}\right)^{k+1}}{k+2}\left\{k+2-y_{0}+\frac{\tau_{0}}{P}\right\}-\frac{\left(y-\frac{\tau_{0}}{P}\right)^{k+2}}{k+2}\right] \\
& \text { for } \quad y_{0} \leq y \leq h_{1} \text {, } \\
& \psi^{(2)}=-\frac{P y^{3}}{6 \mu}+\left(\frac{P h^{2}}{2 \mu}-1\right) y+\left(q+h-\frac{P h^{3}}{3 \mu}\right) \quad \text { for } \quad h_{1} \leq y \leq h,
\end{aligned}
$$

where $P=-\frac{\partial p}{\partial x}$.

As $\tau_{0} \rightarrow 0$ (i.e., $y_{0} \rightarrow 0$ ) and $k=1$, the results obtained agree well with the results of Brasseur et al. 21. The stream function for the case of a power-law fluid (single fluid) is obtained by setting $\mu=1, h_{1}=h$ and $\tau_{0}=0$ in any one of the solutions (3.13). Also the stream function for the Bingham fluid case is obtained by setting $\mu=1, n=1$ and $h_{1}=h$ in (3.13).

The velocity field is given by

$$
\begin{aligned}
& u^{(1)}=\frac{\left(P h_{1}-\tau_{0}\right)^{k+1}-\left(P y-\tau_{0}\right)^{k+1}}{P(k+1)}+\frac{P\left(h^{2}-h_{1}^{2}\right)}{2 \mu}-1, \\
& u_{p}^{(1)}=\frac{\left(P h_{1}-\tau_{0}\right)^{k+1}-\left(P y_{0}-\tau_{0}\right)^{k+1}}{P(k+1)}+\frac{P\left(h^{2}-h_{1}^{2}\right)}{2 \mu}-1, \quad \text { and } \\
& u^{(2)}=-1+\frac{P}{2 \mu}\left(h^{2}-y^{2}\right) .
\end{aligned}
$$

Also we have $\Psi_{y y}^{(1)}=0$ at $y=y_{0}$. Using this condition and (3.13), we get the upper limit of the plug flow region $y_{0}$ as a solution of the equation

$$
\tau_{0}\left(h_{1}^{3}-3 h^{2} h_{1}+2 h^{3}\right)-6 \mu\left(Q-Q_{1}\right) y_{0}=0 .
$$


The interface is also a streamline as seen from the boundary condition (3.10). For a given geometry of the wave and the time-averaged flux $\bar{Q}$, the unknown interface $h_{1}(x)$ is solved from (3.13) using the boundary conditions $\Psi_{1}=q_{1}$ and $\Psi_{2}=q_{2}$. Substituting $\Psi_{1}=q_{1}$ in (3.13a), we get

$$
\begin{aligned}
Q_{1}=\frac{P h_{1}}{2 \mu}\left(h^{2}-h_{1}^{2}\right)+\frac{P^{k}}{k+1} & {\left[h_{1}\left(h_{1}-\frac{\tau_{0}}{P}\right)^{k+1}\right.} \\
& \left.-\frac{\left(y_{0}-\frac{\tau_{0}}{P}\right)^{k+1}}{k+2}\left\{(k+1) y_{0}+\frac{\tau_{0}}{P}\right\}-\frac{\left(h_{1}-\frac{y_{0}}{P}\right)^{k+2}}{k+2}\right]
\end{aligned}
$$

where $Q_{1}=q_{1}+h_{1}$.

For determining $P$ in equation (3.16) we use the continuity of the stream function at the interface given by $\psi_{1}=q_{1}$ at $y=h_{1}$. Hence,

$$
Q_{1}=Q-\frac{P}{6 \mu}\left[h_{1}^{3}-3 h^{2} h_{1}+2 h^{3}\right]
$$

where $Q=q+h$.

Eliminating $P$ from equations (3.16) and (3.17), we get the nonlinear equation governing the interface. The equation for the interface is given by

$$
\begin{aligned}
& \frac{3 h_{1}\left(Q-Q_{1}\right)\left(h^{2}-h_{1}^{2}\right)}{d} \\
& \quad+\frac{1}{(k+1) d^{k}}\left[\frac{h_{1}\left(6 \mu\left(Q-Q_{1}\right) h_{1}-\tau_{0} d\right)^{k+1}}{6 \mu\left(Q-Q_{1}\right)}\right. \\
& \quad-\frac{1}{36 \mu^{2}(k+2)\left(Q-Q_{1}\right)^{2}}\left(6 \mu\left(Q-Q_{1}\right) y_{0}-\tau_{0} d\right)^{k+1}\left\{6 \mu(k+1) y_{0}\left(Q-Q_{1}\right)+\tau_{0} d\right\} \\
& \left.-\frac{1}{36 \mu^{2}(k+2)\left(Q-Q_{1}\right)^{2}}\left(6 \mu\left(Q-Q_{1}\right) h_{1}-y_{0} d\right)^{k+2}\right]-Q_{1}=0,
\end{aligned}
$$

where $d=h_{1}^{3}-3 h^{2} h_{1}+2 h^{3}$.

4. Discussion of the results. As it is difficult to express $q_{1}, h_{1}$ and $P$ explicitly, their values are computed using an iterative procedure. Without loss of generality, we take $y_{0}$ as zero in all the computations involving small values of the yield stress $\tau_{0}$. The interface which is a streamline in the wave frame is determined for different yield stresses with $\alpha=0.7, \phi=0.6, \bar{Q}=0.1, k=3$, and $\mu=0.1$ and is shown in Figure 2 . It is observed that the variation of the interface shape for low-yield stresses gives rise to a thinner peripheral layer in the dilated region. The uniform sinusoidal interface shape is not observed in the figure. From Figure 3, it is found that an increase in the viscosity ratio $\mu$ gives rise to a thinner peripheral layer. From Figure 4 it is noticed that as $k$ increases, the thickness of the peripheral layer increases in the region $0 \leq x \leq 0.6$.

The relationship between $\bar{Q}$ and $\Delta p$ is depicted in Figure 5 for different amplitude ratios when $\mu=0.1, \tau_{0}=0.1, k=3$, and $\alpha=0.7$. We observed that for a given $\Delta p$, the flux $\bar{Q}$ increases with increasing amplitude ratio $\phi$. For a given flux $\bar{Q}$, the pressure rise $\Delta p$ increases with an increased amplitude ratio $\phi$. 


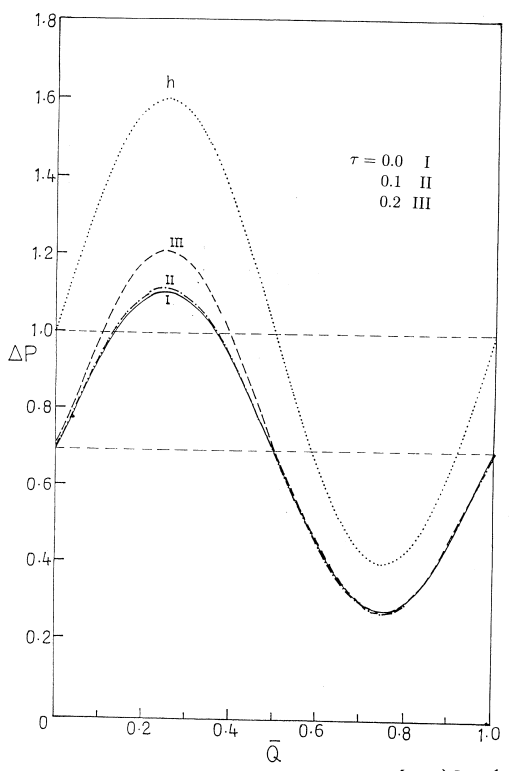

FIG. 2. The shape of the interface for $\alpha=0.7, \phi=0.6, \bar{Q}=$ $0.1, \mu=0.1, k=3$ and for different yield stresses $\tau$

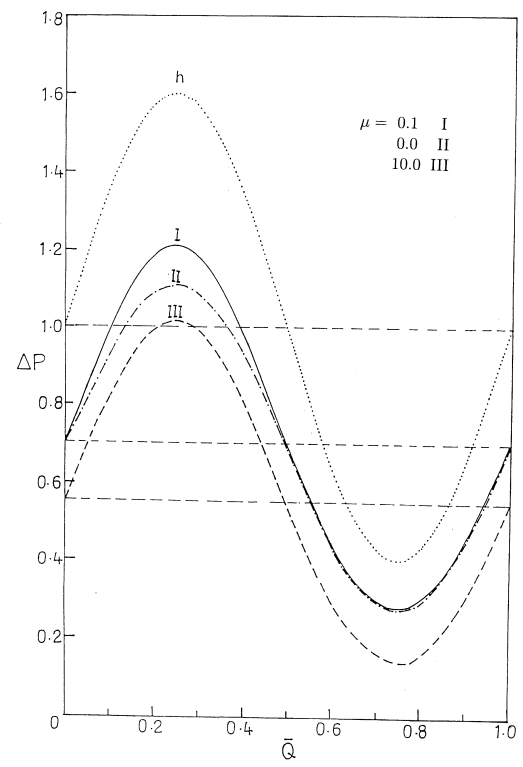

FIG. 3. The shape of the interface for $\alpha=0.6, \phi=0.6, \bar{Q}=$ $0.1, \tau_{0}=0.1$ and for different viscosity ratios $\mu$ 


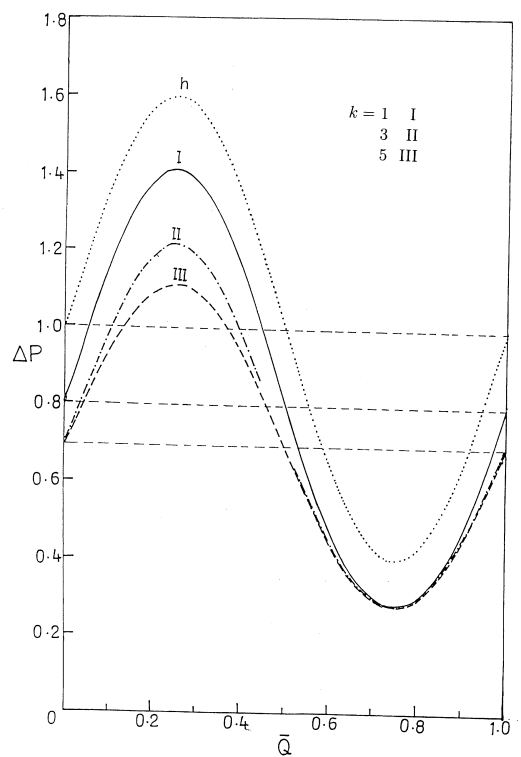

FIG. 4. The shape of the interface for $\alpha=0.7, \phi=0.6, \bar{Q}=$ $0.1, \tau_{0}=0.1$ and for different index values

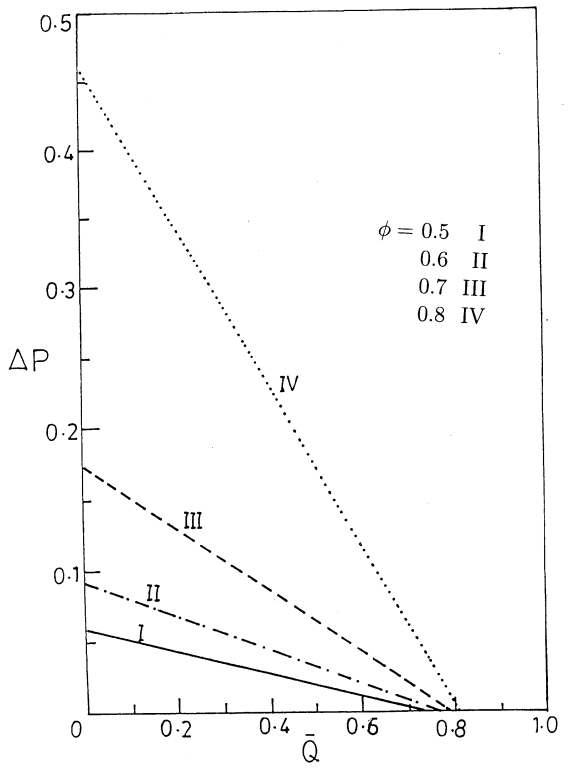

FIG. 5. The variation of $\Delta P$ with $\bar{Q}$ for different $Q$ for $\mu=0.1, \tau_{0}=$ $0.1, k=3$ and $\alpha=0.7$ 


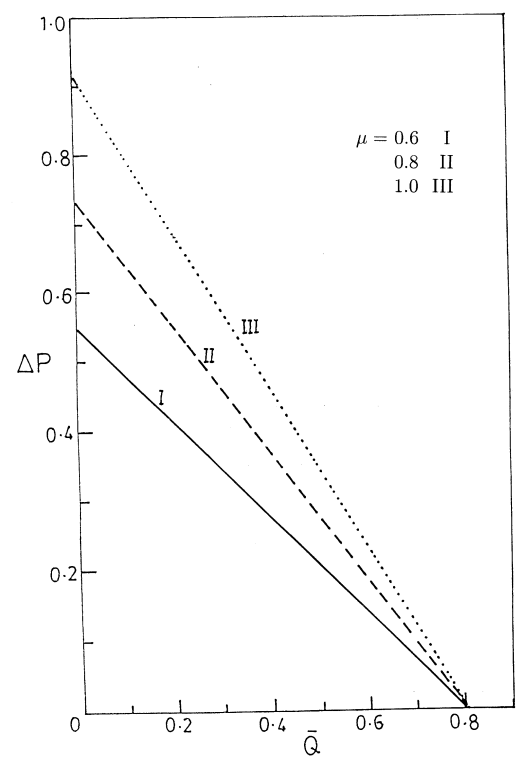

Fig. 6. The variation of $\Delta P$ with $\bar{Q}$ for different $\mu$ for $\alpha=0.7, \phi=$ $0.6, \tau_{0}=0.1$ and $k=3$

The variation of pressure rise with time-averaged flux is calculated and presented in Figure 6 for different values of viscosity ratios $\mu$ with $\alpha=0.7, \phi=0.6, \tau_{0}=0.1$. It is found that for a given $\Delta p$, the flux $\bar{Q}$ depends on $\mu$ and increases with increasing $\mu$. For a given flux $\bar{Q}, \Delta p$ increases with increasing $\mu$.

Acknowledgements. The authors thank the referee and Professor W. Freiberger for constructive comments which led to a definite improvement in the paper.

\section{REFERENCES}

[1] T. W. Latham, Fluid motions in a peristaltic pump, M.S. Thesis, M.I.T., Cambridge, Massachusetts, 1966.

[2] A. H. Shapiro, Pumping and retrograde diffusion in peristaltic waves, Proc. Workshop in Ureteral Reflux in Children (1967), 109-126.

[3] J. C. Burns and T. Parkes, Peristaltic motion, J. Fluid Mech. 29 (1967), 731-743.

[4] A. H. Shapiro, M. Y. Jaffrin and S. L. Weinberg, Peristaltic pumping with log wavelength at low Reynolds number, J. Fluid Mech. 37 (1969), 799-825.

[5] M. Y. Jaffrin, Inertia and streamline curvature on peristaltic pumping, Int. J. Engrg. Sci. 11 (1973), 681-699.

[6] C. Barton and S. Raynor, Peristaltic flow in tubes, Bull. Math. Biophysics 30 (1968), 663-680.

[7] C. C. Yin and Y. C. Fung, Peristaltic waves in circular cylindrical tubes, Trans. ASME J. Appl. Mech. 36 (1969), 579-587.

[8] M. Y. Jaffrin and A. H. Shapiro, Peristaltic pumping, Ann. Rev. Fluid Mech. 3 (1971), 13-36.

[9] J. N. Kapur, Mathematical Models in Biology and Medicine, Affiliated East-West Press Private Limited, New Delhi, 1985. MR0806352 (87k:92001) 
[10] G. W. S. Blair and D. C. Spanner, An Introduction to Amsterdam, Oxford and New York; Elsevier Biorheology Scient. Pub. Co., 1974.

[11] P. Chaturani and R. P. Samy, A study of non-Newtonian aspects of blood flow through stenosed arteries and its application in arterial diseases, J. Biorheology 22 (1985), 521-531.

[12] G. B. Bohme and R. Friedrich, Peristaltic flow of viscoelastic liquids, J. Fluid Mech. 138 (1983), 109-122.

[13] E. F. E. Shehawey and K. S. Mekheimer, Couple-stresses in peristaltic transport of fluids, J. Physics D: Appl. Phys. 27 (1994), 1163-1170.

[14] O. Eytan, A. J. Jaffa and D. Elad, Peristaltic flow in a tapered channel; application to embryo transport within the uterine cavity, J. Medical Engrg. Physics 23 (2001), 473-482.

[15] J. M. Krishnan and K. R. Rajagopal, Review of the uses and modeling of bitumen from ancient to modern times, Appl. Mech. Reviews 56 (2003), 149-214.

[16] J. Málek, J. Nečas, M. Rokyta and M. Rúžička, Weak and Measure-valued Solutions to Evolutionary PDEs, Chapman \& Hall, London, 1996. MR.1409366 (97g:35002)

[17] J. Málek, J. Nečas and K. R. Rajagopal, Global existence of solutions for flows of fluids with pressure and shear dependent viscosities, Appl. Math. Letters 15 (2002), 961-967. MR1925921 (2003g:76032)

[18] J. Málek, K. R. Rajagopal and M. Rúžička, Existence and regularity of solutions and the stability of the rest state for fluids with shear dependent viscosity, Math. Models Methods Appl. Sci. 5 (1995), 789-812. MR 1348587 (96i:76002)

[19] A. N. Alexandrou, T. M. McGilvreay and G. Burgos, Steady Herschel-Bulkley fluid flow in threedimensional expansions, J. Non-Newtonian Fluid Mech. 100 (2001), 77-96.

[20] J. B. Shukla, R. S. Parihar, B. R. P. Rao and S. P. Gupta, Effects of peripheral-layer viscosity on peristaltic transport of a bio-fluid, J. Fluid Mech. 97 (1980), 225-237. MR0566650 (81a:76053)

[21] J. G. Brasseur, S. Corrsin and N. W. Lu, The influence of a peripheral layer of different viscosity on peristaltic pumping with Newtonian fluid, J. Fluid Mech. 174 (1987), 495-519.

[22] A. R. Rao and S. Usha, Peristaltic transport of two immiscible viscous fluids in a circular tube, $J$. Fluid Mech. 298 (1995), 271-285.

[23] S. Usha and A. R. Rao, Peristaltic transport of two-layered power-law fluids, J. Biomech. Engrg. 119 (1997), 483-488.

[24] J. M. Zahm, D. Pierrot, C. Fuchey, J. Levrier, D. Duval, K. G. Lloyd and E. Puchelle, Comparative rheological profile of rate gastric and duodenal gel mucus, Biorheology 26 (1989), 813-822. 